\title{
Exil, Asyl, Flucht
}

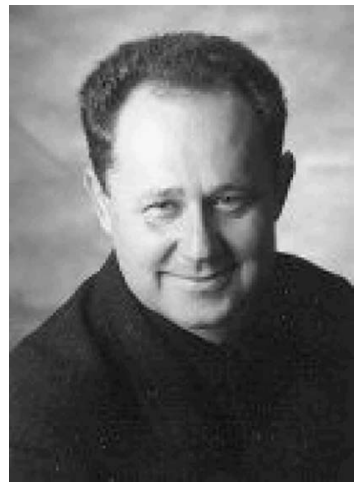

Prof. Dr. med. Manfred Wildner
Was Thomas Mann, Hanna Arendt, Franz Werfel, der Dalai Lama und viele andere mehr gemeinsam haben? Es ist der zumindest zeitweise, durch widrige äußere Umstände ausgelöste Verlust der angestammten Heimat. Ein Leben im Exil: Gründe dafür sind staatlich angeordnete Ausweisungen oder eine freiwillige Flucht vor unerträglichen Zuständen, religiösen, rassischen oder politischen Verfolgungen in der bisherigen Heimat. Sie geben den Anlass für die Suche nach einem schützenden Asyl bzw. eine neue Heimat. Heimat - nur ein geografisch bestimmter Ort? Neben den geografischen Koordinaten sind auch eine Zeitdimension, eine soziale Dimension und eine kulturelle Dimension zu berücksichtigen (Handschuh). Bezogen auf die Zeitdimension ist es gerade die Kindheit, die am stärksten mit den Heimatgefühlen assoziiert zu sein scheint. Vielleicht auch wegen der sozialen Dimension: den engen Verknüpfungen im Netz der Familie, von Freunden und Nachbarn. Bezogen auf den größeren sozialen Rahmen zeigt sich schnell auch eine kulturelle Dimension. Diese prägt Vorlieben und Verhaltensweisen jenseits der individuellen Ausrichtung - einschließlich mancher idealisierender Vorstellungen von Heimat. Dies alles geht bei Flucht oder Exilierung verloren.

Doch ist es nicht auch anders herum, dass durch Exilierung und Flucht fremde Sprachen, Gebräuche und Menschen unerwartet in einem vertrauten heimatlichen Umfeld ankommen? Und so auch für die vordergründig Beheimateten ein vertrautes soziales und kulturelles Umfeld verloren geht und damit Abwehrreaktionen hervorruft? Ein Beispiel dafür? Noch unter dem Eindruck der aus Deutschland während der Unrechtsherrschaft des Nationalsozialismus geflohenen Mitbürger wurde in der neu geordneten Bundesrepublik in $§ 16$ des Grundgesetzes (GG) ein Asylrecht verankert: „Politisch Verfolgte genießen Asylrecht.“ Dieses Recht wurde zunächst nur von Wenigen in Anspruch genommen. Aus den Wenigen wurden durch das Auseinanderbrechen der Sowjetunion und Jugoslawiens Viele. Im Anschluss an das Jahr 1992, welches alleine fast eine halbe Million Asylanträge in Deutschland aufwies, wurde $§ 16$ GG mit dem sogenannten Asylkompromiss ergänzt. Fast möchte man sagen, er wurde aufgehoben: durch den Zusatz, dass eine Einreise aus einem Mitgliedstaat der Europäischen Gemeinschaft oder aus einem anderen sicheren Drittstaat nach Deutschland keine Anwendungsgrundlage des Asylrechts mehr darstellt. Damit blieben bei der geografischen Lage Deutschlands letztlich nur noch die Schiffs- und Flughäfen relevante Außengrenzen.

Verhindern lassen sich Exil und Flucht damit nicht: Menschen brechen weiterhin aus den genannten Gründen in ein besseres, menschenwürdigeres Leben auf, oft genug in Richtung Europa. Auch wenn aufgrund des Asylkompromisses die Anerkennungsquote nach §16a GG nur mehr unter $2 \%$ beträgt, sind die in Deutschland gestellten Anträge doch in einem Drittel bis zur Hälfte der Fälle erfolgreich. Der Grund liegt in einer rechtlichen Alternative, nämlich der Genfer Flüchtlingskonvention (GFK) von 1951, welcher die neu gegründete Bundesrepublik frühzeitig beigetreten ist. Auf dieser Grundlage ist eine Flüchtlingseigenschaft dann gegeben, wenn Menschen aufgrund ihrer Rasse, ihrer Religion, ihrer Nationalität, ihrer Zugehörigkeit zu einer bestimmten sozialen Gruppe oder wegen ihrer politischen Überzeugung in ihrem gewöhnlichen Aufenthaltsstaat verfolgt werden. Zuständig für die Anerkennung ist das Bundesamt für Migration und Flüchtlinge. Mit dieser Anerkennung erwerben Flüchtlinge Rechte, welche in Deutschland durch das Asylbewerberleistungsgesetz (AsylbLG) bzw. europarechtlich durch die Richtlinie 2013/33/EU bestimmt werden. Und sicherlich gibt es auch noch den welfare magnetism, die Anziehungskraft einer hoch erfolgreichen freiheitlichen, sozialen und marktwirtschaftlichen Gesellschaftsordnung. Staatliche Exilierung und politisch begründetes Asylbegehren, Flucht im Sinne der GFK und anderweitig motivierte Migration verschwimmen bisweilen in ihrer Unterscheidung. Folgerichtig steht daher in Ergänzung zum Asylrecht und zum Rechtsrahmen der GFK auch ein Einwanderungsgesetz mit zur Diskussion. Die Bezüge zum Gesundheitswesen? Asylsuchende haben nach $§ 62$ Asylverfahrensgesetz eine ärztliche Untersuchung durch das zuständige Gesundheitsamt auf übertragbare Krankheiten zu dulden. Diese Untersuchung dient sowohl dem Schutz der Asylbewerber bei ihrer Unterbringung in Gemeinschaftsunterkünften wie auch dem Schutz der aufnehmenden Bevölkerung. Eine eventuelle medizinische Versorgung bei akuten Erkrankungen und Schmerzen ist in den $\S \S 4$ und 6 AsylbLG geregelt. Diese sog. Grundleistungen gelten für Personen, die noch nicht 15 Monate ohne wesentliche Unterbrechung im Bundesgebiet Aufenthalt genommen haben. Die Auffangvorschrift des $\$ 6$ AsylbLG stellt eine Öffnungsklausel zur Berücksichtigung konkreter Lebensumstände dar. Die Leistungszuständigkeit liegt zunächst beim örtlichen Träger, also dem Landkreis oder der Kommune. Bei einem Aufenthalt über 15 Monate wird der Versorgungsanspruch an das Leistungsniveau des SGB XII - entsprechend den Regelungen des Sozialhilferechts - angeglichen. Damit besteht Anspruch auf eine Gleichstellung gegenüber den gesetzlich Versicherten [1]. 
Diese Regelungen werfen auch ethische Fragen auf [2-4]. Dabei ist die Frage nach dem Aufenthaltsrecht zur Gefahrenabwehr und zum Schutz von Leib und Leben als Abwehrrecht von der Frage nach dem Umfang und der Höhe von Leistungen als Anspruchsrecht zu differenzieren. Hinzu kommt das Verhältnis der - migrationspolitisch nicht zu relativierenden - vielfältigen Aspekte der Würde zu den nicht minder vielfältigen Aspekten von Gesundheit [5]. Da Ethik eine praktische Wissenschaft ist, ein konkretes Fallbeispiel: Kann eine kurative Therapie bei chronischer Hepatitis C, die pro Tag 1000 EUR kostet und sich über 2-3 Monate erstreckt, nach den Vorgaben des Asylbewerberleistungsgesetzes befürwortet werden? Die anzuwendende Bereichsethik für die Mitarbeiter im öffentlichen (Gesundheits-)Dienst bedeutet im vorliegenden Fall zunächst eine sorgfältige Prüfung im Rahmen der gegebenen Gesetze und Verordnungen. Dabei können Ermessensspielräume unter Berücksichtigung der konkreten Umstände des Einzelfalls genutzt werden. Sollte sich eine weit reichende Folgenschwere der zu treffenden Entscheidung ergeben, z.B. durch ihren Charakter als Präzedenzfall, wären auch die nächsthöheren legitimierten Stellen in die Entscheidungsfindung einzubeziehen.

Spätestens hier knüpft eine tiefer gehende ethische Diskussion an: Wie sind die Ansprüche, die sich aus humanitären Überlegungen sowie aus den allgemeinen und unveräußerlichen Menschenrechten heraus gegenüber der aufnehmenden Gesellschaft ergeben, verglichen mit den gesundheitlichen Versorgungsansprüchen zu werten, die auf dem Prinzip eines Versicherungsverhältnisses beruhen? Erstere werden für Asylbewerber und Flüchtlinge im Rahmen der allgemeinen Steuerpflichten finanziert, letztere zu großen Teilen durch die Mitgliedschaft in einer Solidargemeinschaft oder privaten Krankenversicherung, die auch auf persönlichen Gegenleistungen und damit erworbenen Ansprüchen basiert. Wie steht es um die vergleichende Gerechtigkeit gegenüber anderen bedürftigen Personen („Sans Papiers/ Papierloser“ usw.)? Wie lassen sich die gegebene finanzielle Leistungsfähigkeit, z. B. von Bund, Ländern und Kommunen und auch die gegebene Leistungsfähigkeit und Kapazität des Gesundheitswesens angemessen berücksichtigen? Eine potentielle Zuspitzung dieser ethischen Dilemmata auf gesellschaftlicher Ebene ergibt sich für Ärztinnen und Ärzte durch empfundene Konflikte mit der individualmedizinischen Berufsethik [6]. Dabei ist das medizinisch Machbare allein ohnehin nicht mehr das ausschlaggebende Kriterium für eine Gesellschaft, die sich mit gemeinsamem Bundesausschuss, IQWIG u.a.m. in intensiven Diskussionsprozessen selbst Einschränkungen auferlegt. So spielt im Bereich der Sozialgesetzbücher die Frage nach vertretbaren Kosten durchaus eine Rolle (Wirtschaftlichkeitsgebot, Erlaubnisvorbehalt u.a.m.). Zusätzliche Aspekte ergeben sich aus supranationalen europarechtlichen Regelungen. So wird bspw. Personen aus einem EU-Mitgliedstaat, welche eine notwendige Behandlung in ihrem Heimatland nicht oder nicht rechtzeitig erhalten können, eine solche Behandlung in einem anderen EUMitgliedstaat nur solange zugestanden (Richtlinie 2011/24/EU), wie dies nicht das Gesundheitssystem des aufnehmenden EUMitgliedstaates überfordert [7]. Der Ring für informierte und engagierte Diskussionen und so manchen Schlagabtausch über das Verhältnis von Möglichem und Machbarem, von kluger Außenpolitik und kluger Innenpolitik, von Individuum und Gesellschaft, von Ausgleich und Differenz ist hier freigegeben. Auf die zentrale Rolle der Arbeitsaufnahme als Einstieg in die Solidargemeinschaft der gesetzlichen Krankenversicherung sei für diese Diskussion explizit hingewiesen.
Zur Informationsgrundlage der vielfältigen gesellschaftlichen Entscheidungs- und Selbstfindungsprozesse auch im Zusammenhang mit der aktuellen Flüchtlingsdiskussion wollen die Beiträge und das Gasteditorial dieser Ausgabe beitragen. Sie behandeln Themen wie die Nicht-Inanspruchnahme von Leistungen durch Menschen mit Migrationshintergrund, das Erleben der Gesundheitsversorgung in der Schweiz durch Migranten aus dem Kosovo, Familien mit Migrationshintergrund als Zielgruppe für Frühe Hilfen, Charakteristika primärärztlicher Versorgung von Patienten außerhalb regulärer Sprechstundenzeiten, die Qualität der Versorgung in Deutschland sowie den Umgang mit Wartezeiten im internationalen Vergleich. Der CME-Fortbildungsbeitrag greift das dabei auch wichtige Thema Vektor-übertragener Erkrankungen auf. Eine besondere Freude ist wieder der Mehrwert eines Heftschwerpunktes für Sie als Leser, diesmal die sprachfreie Intelligenzdiagnostik zur Erhebung kognitiver Fähigkeiten und zur Prävention von Entwicklungsrisiken, ebenfalls mit Bezug zu Migration - siehe dazu auch das zugehörige weitere Gasteditorial von Daseking und Petermann.

Antworten auf Fragen zur Leistungsgewährung bei beschränkten Ressourcen sind unbequem und wohl nie für alle Seiten befriedigend zu lösen. Dieses Abwägen zwischen einem mehr oder weniger an Ungenügen ist der Kern eines ethischen Dilemmas. Hier unterscheiden sich die Fragen im Bereich des Asyl- bzw. Flüchtlingsrechtes nicht grundsätzlich von den allgemeinen Fragen der gesundheitlichen Versorgung in einer freiheitlichen, gleichzeitig sozialen und solidarischen Gesellschaft. Ein den komplexen Sachverhalten entsprechender, ausreichend differenzierter Diskurs ist essentiell. Letztlich geht es in diesem mühevollen Ringen darum, uns selbst und andere Menschen mit unseren räumlichen, zeitlichen, sozialen und kulturellen Dimensionen und Verantwortungssphären in der konkreten Welt zu beheimaten. Dabei sollten wir das Risiko eines Nicht-beheimatet-Seins nicht nur bei exilierten Personen und Flüchtlingen im Außenbereich unserer Gesellschaft ansiedeln, sondern auch als zumindest zeitweise biografische Realität für viele Mitglieder im Inneren einer Gesellschaft wahrnehmen. Womit deutlich wird, um was es in diesem gesellschaftlichen Ringen um die Wertetrias von Solidarität, Inklusion und Humanität auch geht: dass wir unsere soziale und kulturelle Heimat bewahren bzw. gestaltend neu gewinnen und - vielleicht sogar mit einer globalen Komponente - in Richtung einer menschenwürdigen und lebenswerten Zukunft konstruktiv weiterentwickeln.

\section{Literatur}

1 Friesacher A. Medizinische Versorgung von Asylbewerbern. KVB Forum 2015; 7-8: 28-29

2 Schröder-Bäck P. Ethische Prinzipien für die Public Health Praxis. Frankfurt am Main: Campus Verlag; 2014

3 Kostka U. Lampedusa in Berlin. Zum ethisch verantwortlichen Umgang mit Flüchtlingen und ihrer Gesundheit. Public Health Forum 2015; 23: 91-92

4 Lammers $H$. Gesundheitsversorgung von Flüchtlingen - zur gesetzlich festgelegten Zweiklassenversorgung. Public Health Forum 2015; 23: 93-94

5 Deutscher Bundestag. Antwort der Bundesregierung auf die kleine Anfrage „Gesundheitliche Versorgung nach dem Asylbewerberleistungsgesetz“. Drucksache 18/2184 vom 22.07.2014

6 Bundesärztekammer. Stellungnahme der zentralen Kommission zur Wahrung ethischer Grundsätze in der Medizin und ihren Grenzgebieten (Zentrale Ethikkommission) bei der Bundesärztekammer „Versorgung von nicht-regulär kranken versicherten Patienten mit Migrationshintergrund“. Deutsches Ärzteblatt 2013; 110: A899-A903

7 Europäische Union. Richtlinie 2011/24/EU des Europäischen Parlaments und des Rates vom 9. März 2011 über die Ausübung der Patientenrechte in der grenzüberschreitenden Gesundheitsversorgung URL: http://eur-lex.europa.eu/legal-content/DE/TXT/?uri = celex:32011L0024 (Zugriff am 29.07.2015) 